\title{
Association of Superior Oblique Muscle Volumes with the Presence or Absence of the Trochlear Nerve on High- Resolution MR Imaging in Congenital Superior Oblique Palsy
}

H.K. Yang, D.S. Lee, J.H. Kim, and J.-M. Hwang

\begin{abstract}
BACKGROUND AND PURPOSE: Congenital superior oblique palsy is known to relate to trochlear nerve absence and a variable degree of superior oblique muscle hypoplasia. The purpose of this study was to determine whether superior oblique muscle volume predicts trochlear nerve absence in congenital superior oblique palsy.
\end{abstract}

MATERIALS AND METHODS: A retrospective study of high-resolution MR imaging to evaluate the presence of the trochlear nerve and to measure superior oblique muscle areas and volumes with the image analysis tools of a PACS was performed in 128 consecutive patients with unilateral congenital superior oblique palsy and 34 age-matched healthy controls.

RESULTS: Of the 128 patients with congenital superior oblique palsy, 88 had an ipsilateral trochlear nerve absence (absent group) and 40 had both trochlear nerves (present group). In patients with congenital superior oblique palsy, the paretic side superior oblique muscle volume was significantly smaller compared with the normal side only in the absent group $(P<.001)$. The left and right side superior oblique muscle volumes were not significantly different in controls $(P=.750)$, and the paretic and normal side superior oblique muscle volumes were not significantly different in the present group $(P=.536)$. The cutoff value of the paretic/normal side superior oblique muscle volume ratio for diagnosing trochlear nerve absence was $\leq 0.75$ (sensitivity $98.9 \%$, specificity $95.0 \%$ ) in patients with congenital superior oblique palsy.

CONCLUSIONS: The ratio of paretic/normal side superior oblique muscle area and volume has an excellent predictability in diagnosing trochlear nerve absence in congenital superior oblique palsy.

ABBREVIATIONS: $\mathrm{AUC}=$ area under the receiver operating characteristic curve; $\mathrm{SO}=$ superior oblique muscle; SOP $=$ superior oblique palsy

A dvanced imaging modalities and functional anatomy have helped us understand the etiology and pathophysiology of superior oblique palsy (SOP). ${ }^{1-12}$ Previous studies of SOP by us-

Received August 30, 2014; accepted after revision October 26.

From the Departments of Ophthalmology (H.K.Y., D.S.L., J.-M.H.) and Radiology (J.H.K.), Seoul National University College of Medicine, Seoul National University Bundang Hospital, Seongnam, Korea.

H.K. Yang and D.S. Lee contributed equally to this work.

This work was supported by the Basic Science Research Program through the National Research Foundation of Korea funded by the Ministry of Education, Science and Technology (2013R1A1A2010606) and the Interdisciplinary Research Initiatives Program by College of Engineering and College of Medicine, Seoul National University (2012).

Please address correspondence to Jeong-Min Hwang, MD, Department of Ophthalmology, Seoul National University College of Medicine, Seoul National University Bundang Hospital, 166 Gumiro, Bundang-gu, Seongnam, Gyeonggi-do 463-707, Korea; e-mail: hjm@snu.ac.kr; and Jae Hyoung Kim, MD, Department of Radiology, Seoul National University College of Medicine, Seoul National University Bundang Hospital, 166 Gumiro, Bundang-gu, Seongnam, Gyeonggi-do 463-707, Korea; e-mail: jaehkim@snubh.org

- Indicates open access to non-subscribers at www.ajnr.org

http://dx.doi.org/10.3174/ajnr.A4202 ing MR imaging have consistently shown a variable degree of hypoplasia of the paretic superior oblique muscle $(\mathrm{SO})$ and variable trochlear nerve absence. ${ }^{1-5}$ In our recent study of patients with congenital SOP, $73 \%$ had ipsilateral trochlear nerve absence and a variable degree of SO hypoplasia, while the remaining $27 \%$ had a normal-appearing SO and trochlear nerve on both sides, suggesting a different etiology. ${ }^{3}$

Consistent identification of the trochlear nerve, however, requires high-resolution MR imaging with at least a $3 \mathrm{~T}$ system, $0.25-\mathrm{mm}$ section thickness, a scanning plane set to an oblique axial direction parallel to the course of the trochlear nerve, and experienced interpretation. Thus, it may not be practical to perform high-resolution MR imaging in many institutions. On the other hand, the cross-sectional area of the SO is easily determined in the coronal plane of the most commonly used orbital MR imaging sequences, or CT. Therefore, it would be useful to be able to predict trochlear nerve absence by the degree of SO hypoplasia. Nevertheless, a quantified volumetric evaluation of the $\mathrm{SO}$ and its relationship to the presence or absence of the trochlear nerve has 

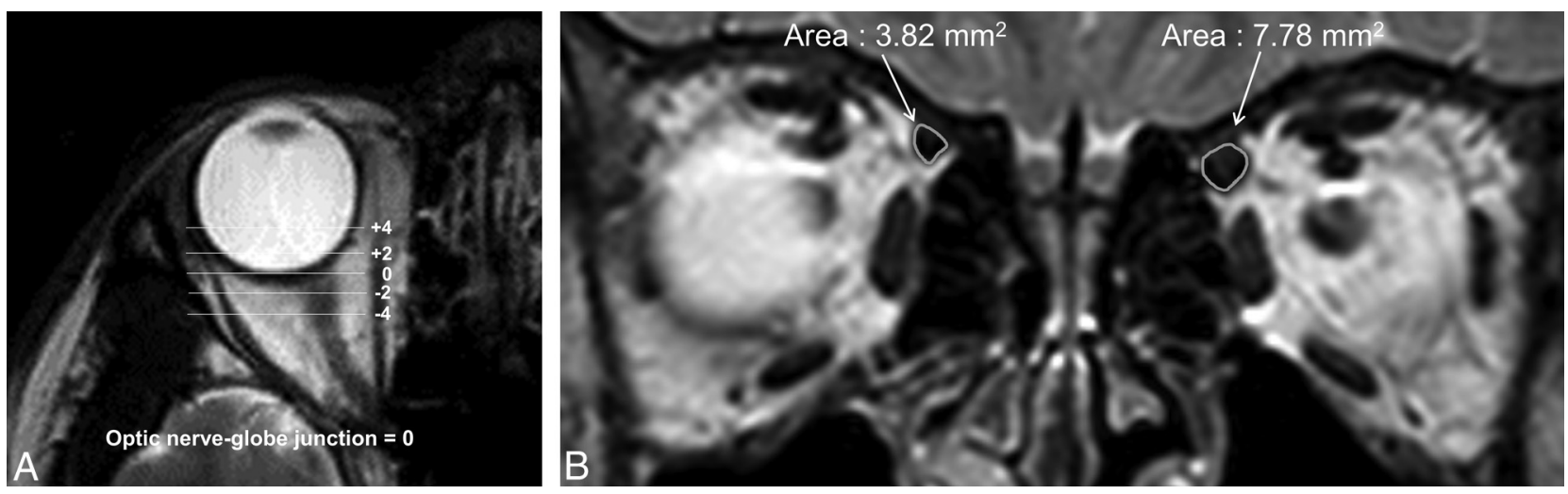

FIG 1. MR imaging in a patient with right superior oblique muscle palsy. A, The optic nerve-globe junction was defined as the standard plane (plane "0"), and SO areas were measured in the coronal sections of 5 contiguous planes, including the standard plane and planes that were 2 and $4 \mathrm{~mm}$ anterior or posterior to the standard plane. B, T2-weighted coronal image of the orbit and SO. The right SO is hypoplastic compared with the left. The area surrounded by the curvilinear line was measured in 5 different planes, by using DTU-710 (Wacom) and PACS software, which provides automatic measurements for area. The volume of the SO was defined as the sum of SO areas at the 5 planes multiplied by 2 mm.

not yet been determined, to our knowledge. In this study, we measured the cross-sectional areas and volume of the SO by using MR imaging and determined the ability of SO volumetry to predict trochlear nerve absence in patients with congenital SOP.

\section{MATERIALS AND METHODS}

A retrospective review of medical records was performed for 128 consecutive patients diagnosed with unilateral congenital SOP and 34 age-matched controls who had undergone thin-section MR imaging at the brain stem level at Seoul National University Bundang Hospital between August 2009 and June 2012. Patients were included if they showed the typical signs of congenital SOP, including apparent underdepression and overelevation in adduction on the affected side, positive head tilt test results, large fusional amplitudes of vertical deviation, and a history or photographic evidence of long-standing strabismus or anomalous head posture dating to infancy. Patients who had primary overaction of the inferior oblique muscle on the affected side, any evidence of acquired disease, a history of head or ocular trauma, or other potential causes, such as plagiocephaly, skew deviation, myasthenia gravis, or the ocular tilt reactions, were excluded. Subjects without strabismus and patients with simple horizontal strabismus without oblique muscle dysfunction were enrolled as the control group. Patient characteristics noted included sex, age at examination, and laterality of the paretic side. Approval to conduct this study was obtained from the institutional review board of Seoul National University Bundang Hospital.

\section{Superior Oblique Muscle Volumetry}

MR imaging was performed by using a 3T system (Intera Achieva; Philips Healthcare, Best, the Netherlands) with an 8-channel sensitivity encoding head coil, initially with T2-weighted imaging of the entire brain and the orbit and subsequently with high-resolution imaging of cranial nerves and the brain stem. ${ }^{3}$ The initial orbital imaging to evaluate the extraocular muscles was performed, without the use of visual targets for fixation, with the turbo spin-echo technique. The sequence protocol was same as that in our previous studies. . $^{3,4}$

The SO area was measured on T2-weighted coronal images of the orbit. The optic nerve-globe junction was defined as the standard plane (plane " 0 "), and SO areas were measured in 5 contiguous coronal image planes, including the standard plane and the planes that were $2 \mathrm{~mm}$ and $4 \mathrm{~mm}$ anterior or posterior to the standard plane (Fig 1). We measured the area of the SO by using DTU-710 (Wacom, Saitama, Japan) and PACS software, which provides automatic measurement for area. The volume of the $\mathrm{SO}$ was defined as the sum of $\mathrm{SO}$ areas at the 5 planes multiplied by $2 \mathrm{~mm}$. Measurement of the SO area on the 5 planes, SO volume, ratios of paretic/normal side SO area, and volume (left/ right for controls, paretic/nonparetic for those with SOP) were investigated. Three individual interpreters measured the data, blinded to the patient's clinical status and trochlear nerve image. Measurements were repeated 3 times for each examiner, and the average of the 9 values was used for the comprehensive analysis.

\section{Statistical Analyses}

Statistical analyses were performed by using SPSS software for Windows, Version 18.0 (IBM, Armonk, New York). We compared groups by using the ANOVA with a Bonferroni post hoc test and the Pearson $\chi^{2}$ test. The sensitivity and the specificity of SO area and $\mathrm{SO}$ volume for predicting trochlear nerve absence were determined by receiver operating characteristic curves and area under the receiver operating characteristic curves (AUCs). The interobserver intraclass correlation coefficients and their 95\% confidence intervals were calculated among the measurements.

\section{RESULTS}

\section{Patient Characteristics}

Of the 128 consecutive patients diagnosed with unilateral congenital SOP (88 patients with an ipsilateral trochlear nerve absence and 40 patients with normal anatomy of the trochlear nerve on both sides), 3 were excluded due to poor quality of MR images, with obscured SO margins in the midst of surrounding soft tissues and motion artifacts, or with an SO tendon extending more posteriorly than the optic nerve-globe junction in both eyes. Hence, MR imaging measurements of the SO were performed on 125 patients with unilateral congenital SOP and 34 age-matched healthy controls. Of the 125 patients with unilateral congenital 
Patient characteristics and the superior oblique muscle area and volume measured in controls and patients with congenital superior oblique palsy without (absent group) and with (present group) an ipsilateral trochlear nerve

\begin{tabular}{|c|c|c|c|c|}
\hline & $\begin{array}{l}\text { Control } \\
(n=34)\end{array}$ & $\begin{array}{l}\text { Absent Group } \\
\qquad(n=87)\end{array}$ & $\begin{array}{l}\text { Present Group } \\
\qquad(n=38)\end{array}$ & $P$ Value \\
\hline Age at time of imaging (yr) & $16.1 \pm 20.7(1-69)$ & $15.2 \pm 17.9(1-64)$ & $11.5 \pm 13.0(1-58)$ & $.896^{\mathrm{a}}$ \\
\hline Age of onset (yr) & & $10.8 \pm 16.9(0-63)$ & $7.7 \pm 13.4(0-58)$ & $.317^{\mathrm{b}}$ \\
\hline Interval between onset and imaging (yr) & & $3.8 \pm 6.4(0-44)$ & $3.8 \pm 4.8(0-23)$ & $.967^{\mathrm{b}}$ \\
\hline Male sex & $16(47.0 \%)$ & $55(63.2 \%)$ & $21(55.3 \%)$ & $.283^{c}$ \\
\hline \multicolumn{5}{|l|}{ Paretic side } \\
\hline Right & & $48(55.2 \%)$ & $17(44.7 \%)$ & $.085^{c}$ \\
\hline Left & & $39(44.8 \%)$ & $21(55.3 \%)$ & \\
\hline SO area "O" (P) & $4.58 \pm 1.05(2.68-6.89)$ & $2.12 \pm 0.96(0.34-4.59)$ & $5.01 \pm 1.02(3.39-7.78)$ & $<.001^{\mathrm{a}}$ \\
\hline SO area “O” (N) & $4.43 \pm 1.23(2.41-8.53)$ & $5.34 \pm 1.31(2.17-10.45)$ & $4.87 \pm 1.11(3.11-8.17)$ & $.001^{\mathrm{a}}$ \\
\hline $\mathrm{SO}$ area "0" ratio $(\mathrm{P} / \mathrm{N})^{\mathrm{d}}$ & $1.06 \pm 0.18(0.78-1.42)$ & $0.40 \pm 0.15(0.05-0.75)$ & $1.04 \pm 0.10(0.86-1.21)$ & $<.001^{\mathrm{a}}$ \\
\hline SO volume $(P)^{e}$ & $41.57 \pm 8.15$ (27.03-61.17) & $21.53 \pm 8.69(3.92-40.12)$ & $46.99 \pm 9.50(28.52-75.99)$ & $<.001^{\mathrm{a}}$ \\
\hline SO volume $(\mathrm{N})^{\mathrm{f}}$ & $40.94 \pm 9.87(24.16-73.10)$ & $49.31 \pm 11.15(27.93-94.04)$ & $46.76 \pm 9.60(30.95-76.00)$ & $.001^{\mathrm{a}}$ \\
\hline SO volume ratio $(P / N)^{d}$ & $1.03 \pm 0.15(0.84-1.54)$ & $0.44 \pm 0.15(0.07-0.74)$ & $1.03 \pm 0.10(0.79-1.20)$ & $<.001^{\mathrm{a}}$ \\
\hline
\end{tabular}

Note:- P indicates paretic side; N, normal side; "0", measured at the optic nerve-globe junction (standard plane).

a $P$ value by ANOVA.

${ }^{\mathrm{b}} P$ value by independent $t$ test

c $P$ value by Pearson $\chi^{2}$.

${ }^{\mathrm{d}}$ Left-to-right ratio for controls and paretic side to normal side ratio for patients with congenital SOP in the absent and present group.

e Left side for controls.

${ }^{f}$ Right side for controls.

SOP, 87 (69.6\%) were found with an ipsilateral trochlear nerve absence (absent group) and 38 (30.4\%) had normal anatomy of the trochlear nerve on both sides (present group). Controls without SOP had normal anatomic features of the trochlear nerves on both sides (control group). The mean age and sex distribution were not significantly different among the groups $(P=.896,0.283$, respectively). There was no significant difference in the laterality of SOP between the absent and present group $(P=.085)$ (Table).

\section{Superior Oblique Muscle Area and Volume}

In patients with congenital SOP with an absent trochlear nerve, the paretic side SO areas at all 5 contiguous coronal image planes and $\mathrm{SO}$ volume were significantly smaller compared with the normal side SO $(P<.001$ by a paired $t$ test). However, there was no significant difference between the paretic and normal side SO areas and volume in the present group $(P=.536)$, and there was no difference in both sides of SO areas and volume in controls $(P=.750)$. The paretic/normal side ratios of SO areas and SO volume were significantly smaller in the absent group compared with other groups $(P<.001)$ (Table and Fig 2$)$. The calculated interobserver intraclass correlation coefficient of the measured SO area was 0.770 (95\% CI, 0.747-0.790, $P<.001)$.

The paretic side $\mathrm{SO}$ areas in the absent group were smaller than those of the present group and those of the left sides in the controls in all 5 planes $(P<.001$ by ANOVA, post hoc Bonferroni correction). The paretic side $\mathrm{SO}$ areas in the present group were not significantly different from those of the left sides in the controls $(P>.05$ for all 5 planes). The normal side SO areas and volume in congenital SOP were larger than those in controls $(P<$ .001 by ANOVA), but those of the absent group and present group did not show a significant difference $(P>.05$ by ANOVA, post hoc Bonferroni correction).

\section{Predictability of Trochlear Nerve Absence by SO Area and Volume}

Among the receiver operating characteristic curves for predicting trochlear nerve absence (paretic side SO areas, paretic side SO

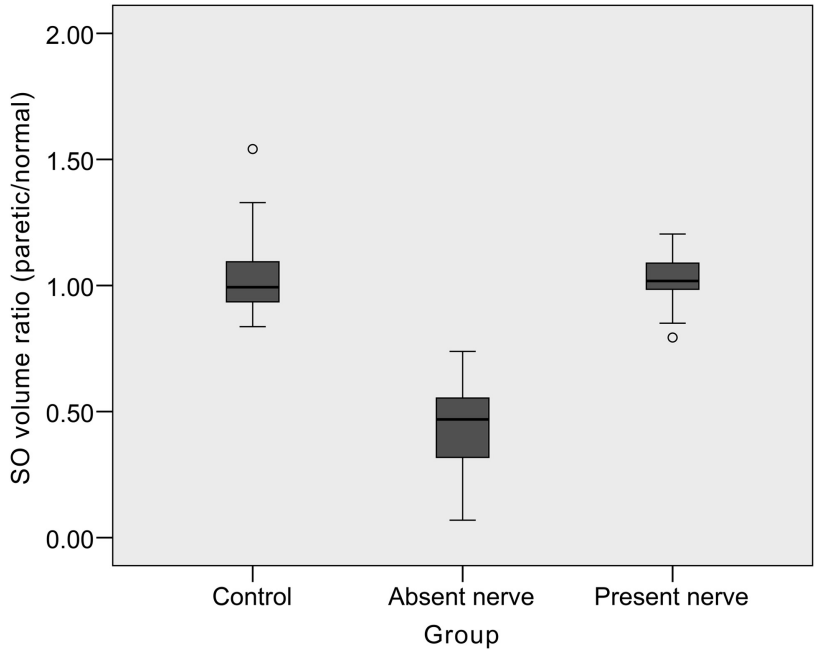

FIG 2. Boxplots of the superior oblique muscle volume ratio of the paretic-to-normal side in patients with congenital superior oblique palsy without (absent group) and with (present group) an ipsilateral trochlear nerve compared with controls. The SO volume ratio was significantly smaller in the absent group $(P<.001)$ compared with controls. There was no significant difference of the $\mathrm{SO}$ volume ratio between controls and the present group.

volume, ratios of paretic/normal side SO areas and volume), AUC was largest for the paretic/normal side ratio of SO volume and SO area at the optic nerve-globe junction (AUC $>0.950$ ) (Fig 3). The cutoff value of the ratio of paretic/normal side SO volume and SO area at the optic nerve-globe junction for diagnosing trochlear nerve absence was $\leq 0.75$ (sensitivity $98.9 \%$, specificity $95.0 \%$ ) in patients with congenital SOP.

\section{DISCUSSION}

In this study, a strong relation was found between the quantitative evaluation of SO volume and the presence or absence of the trochlear nerve in patients with clinically diagnosed congenital SOP. The main findings are as follows: First, the cross-sectional area and volume of the paretic side SO were significantly smaller com- 

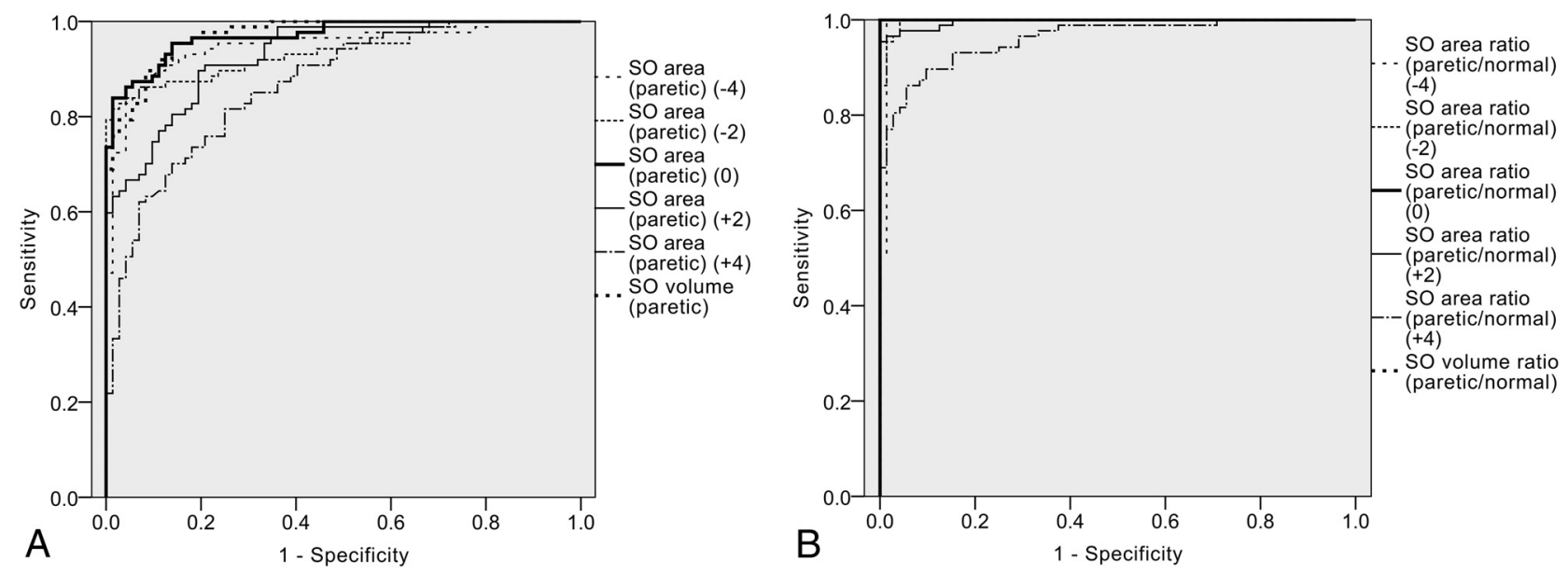

FIG 3. Receiver operating characteristic curves of paretic side SO areas, SO volume, paretic/normal side ratios of the SO area and SO volume for predicting trochlear nerve absence. The area under the receiver operating characteristic curves was largest for the paretic/normal side ratios of SO volume and SO area at the optic nerve-globe junction, with an AUC value of $>0.950$. AUCs of the paretic side SO areas were much smaller at the anterior planes $(+4,+2)$ compared with the standard plane $(0)$ or posterior planes $(-2,-4)$.

pared with the normal side in the absent group of congenital SOP. Second, the ratio of paretic/normal side SO volume and area near the optic nerve-globe junction was $\leq 0.75$ in patients with SOP without the trochlear nerve. Finally, SO area and volume of the contralateral normal side were larger in both groups of patients with congenital SOP than in controls.

Hypoplasia of the ipsilateral SO has been the major finding on MR imaging of patients with congenital or idiopathic SOP. ${ }^{1-5}$ However, the extent of SO hypoplasia compared with the contralateral side varied widely from $0 \%$ to $100 \%$ in previous reports. ${ }^{1-3}$ Sato ${ }^{1}$ stated that in congenital SOP, there were 2 peaks in the distribution of the percentage volume of muscle size compared with the contralateral normal side: one between $20 \%$ and $40 \%$ and the other between $90 \%$ and $100 \%$. These 2 groups correspond well to the absent group and present group of congenital SOP in our study because the mean ratio of paretic/normal side SO volume was $43 \%$ in the absent group and $103 \%$ in the present group - that is, the paretic side SO volume was abnormally small only in the patients with an absent trochlear nerve. As we have demonstrated in our previous study, the 2 groups classified by the absence or presence of the trochlear nerve in congenital SOP account for the 2 main etiologies of congenital SOP. ${ }^{3}$ The first is based on abnormalities of the muscle and its innervations associated with SO hypoplasia and trochlear nerve absence and the other is based on the pathologic features of the tendon or heterotopic muscle pulleys. ${ }^{1,13}$

The ratio of paretic/normal side SO volume and SO area at the optic nerve-globe junction demonstrated excellent predictability for trochlear nerve absence. On the basis of the quantitative results of our study, we may expect that patients with congenital SOP with a significant SO hypoplasia of $\leq 75 \%$ compared with the contralateral normal side are likely to have trochlear nerve absence. As the coronal image plane moved anterior from the optic nerve-globe junction (plane $+2,+4$ ), however, the predictability of the SO area and the ratio of paretic/normal side SO area significantly decreased compared with the optic nerve-globe junction (plane " 0 ") or posterior planes (plane -2, -4) (Fig 3) - that is, SO hypoplasia of the paretic side compared with the normal side was less apparent in the anterior planes. This difference can be inferred from the fact that the anterior part of the SO ends in a rounded tendon, which acts in a fibrocartilaginous pulley attached to the trochlear fovea of the frontal bone. On the other hand, the predictability of the posterior planes $(-2,-4)$ was also lower than that of the optic nerve-globe junction, probably owing to the fusiform structure of the SO. Considering the individual variations in orbital depth and position of the SO muscle/tendon transition, the best plane for evaluating SO hypoplasia should be adjusted to the image plane having the greatest cross-sectional area of the SO. ${ }^{14}$ Previous studies have shown that despite the individual variation, the coronal section at the nearest location posterior to the globe and optic nerve junction may be suitable for evaluating hypoplasia of the extraocular muscles. ${ }^{2,7}$ This finding was consistent with our results, because SO hypoplasia and associated trochlear nerve absence were best predicted by the crosssectional area of the SO near the optic nerve-globe junction. The SO volume and the sum of the SO areas at 5 planes also demonstrated good predictability of SO hypoplasia and trochlear nerve absence. However, the cross-sectional area of the SO in the plane nearest the optic nerve-globe junction was sufficient for this purpose.

The SO area and volume of the contralateral normal side were larger in patients with congenital SOP than in controls. Although it has been reported that the average maximum SO cross-section for the fellow eyes was not significantly different from those in healthy controls, ${ }^{14}$ more recent reports showed a larger size and supernormal contractility of the normal contralateral SO compared with that in controls in patients with SOP. ${ }^{7}$ Contralateral SO hypertrophy corresponds to the clinical finding of contralateral overdepression in adduction and may be suggestive of an adaptive change, change of fiber type distribution, or innervational change. ${ }^{7}$ On the other hand, because we did not control fixation during imaging, variable positions of the eyeball could have affected the muscle size. The normal fellow eye of patients with congenital SOP may show a relatively downward displacement compared with that in healthy controls, and downward dis- 
placement leads to a larger cross-section of the SO and inferior rectus muscle. ${ }^{14}$

The results of our study should be limited to congenital SOP and cannot be applied to acquired or idiopathic SOP. Acquired SOP may also show variable atrophy of the SO muscle, as in the absent group of congenital SOP. ${ }^{15}$ A significant volume loss of the SO muscles in acquired trochlear nerve palsies has also been reported, and the nerve may also "regress" and die back, becoming severely hypoplastic. ${ }^{16}$ The trochlear nerve may regress or become hypoplastic after perinatal injury or, in older patients, from diabetes or a developing small mass along the tentorial incisura. ${ }^{17}$ Thus, just because the volume of the SO is significantly decreased, we cannot conclude that the etiology is from nerve absence. In addition, variable degrees of SO muscle atrophy can be found in both congenital and acquired cases with no significant difference in the appearance of the SO muscle between acquired and congenital SOP groups. ${ }^{15}$ Moreover, the Bielschowsky head tilt test is not specific and may lead to erroneous results. ${ }^{17}$ The presence of large vertical fusional amplitudes does not necessarily imply a congenital etiology because vertical fusional vergence may increase in adults within weeks or months after an acquired vertical strabismus. ${ }^{17}$ Therefore, the diagnosis of congenital or acquired SO palsy cannot be concluded solely by imaging or ocular motor examination, but a thorough history and clinical examination are necessary. In these cases, the clinical features are quite different, such as the age of onset, facial asymmetry, and trochlear nerve status. Congenital SOP would be diagnosed mostly in early childhood with significant head tilt or with facial asymmetry in adulthood. In patients with severe SO muscle atrophy, trochlear nerve imaging with high-resolution MR imaging revealing an absent nerve would favor a congenital etiology, while a relatively normal trochlear nerve would suggest acquired SOP. ${ }^{3}$

There are certain limitations to our study. First, this is a retrospective study, not free of unintended confounding issues and a bias toward selecting severely affected patients in a tertiary referral center. Second, patients were excluded if they had poor-quality MR images: motion artifacts, obscured SO margins, or SO tendons extending more posteriorly than the optic nerve-globe junction, applicable to 3 patients of the total 162 study participants. Third, variable positions of the eyeball could have affected the muscle size. MR imaging sequences with higher resolutions by using fixation targets during imaging could overcome these problems.

\section{CONCLUSIONS}

The ratio of paretic/normal side SO area near the optic nerveglobe junction and SO volume has an excellent predictability for diagnosing trochlear nerve absence in congenital SOP. SO hypoplasia of $\leq 75 \%$ compared with the contralateral normal side suggests trochlear nerve absence as the etiology of congenital SOP.
Disclosures: Jeong-Min Hwang—RELATED: Grant: Basic Science Research Program through the National Research Foundation of Korea funded by the Ministry of Education, Science and Technology (2013R1A1A2010606) and the Interdisciplinary Research Initiatives Program by College of Engineering and College of Medicine, Seoul National University (2012).

\section{REFERENCES}

1. Sato M. Magnetic resonance imaging and tendon anomaly associated with congenital superior oblique palsy. Am J Ophthalmol 1999; 127:379-87

2. Uchiyama E, Matsuo T, Imai S, et al. Paretic side/normal side ratios of cross-sectional areas of the superior oblique muscle vary largely in idiopathic superior oblique palsy. Am J Ophthalmol 2010;149: 508-12

3. Yang HK, Kim JH, Hwang JM. Congenital superior oblique palsy and trochlear nerve absence: a clinical and radiological study. Ophthalmology 2012;119:170-77

4. Kim JH, Hwang JM. Absence of the trochlear nerve in patients with superior oblique hypoplasia. Ophthalmology 2010;117:2208-13.e1-2

5. Kim JH, Hwang JM. MR imaging of familial superior oblique hypoplasia. Br J Ophthalmol 2010;94:346-50

6. Choi BS, Kim JH, Jung C, et al. High-resolution 3D MR imaging of the trochlear nerve. AJNR Am J Neuroradiol 2010;31:1076-79

7. Kono R, Demer JL. Magnetic resonance imaging of the functional anatomy of the inferior oblique muscle in superior oblique palsy. Ophthalmology 2003;110:1219-29

8. Jiang L, Demer JL. Magnetic resonance imaging of the functional anatomy of the inferior rectus muscle in superior oblique muscle palsy. Ophthalmology 2008;115:2079-86

9. Clark RA, Demer JL. Enhanced vertical rectus contractility by magnetic resonance imaging in superior oblique palsy. Arch Ophthalmol 2011;129:904-08

10. Demer JL, Kung J, Clark RA. Functional imaging of human extraocular muscles in head tilt dependent hypertropia. Invest Ophthalmol Vis Sci 2011;52:3023-31

11. Chan TK, Demer JL. Clinical features of congenital absence of the superior oblique muscle as demonstrated by orbital imaging. $J A A$ POS 1999;3:143-50

12. Kono R, Okanobu H, Ohtsuki H, et al. Absence of relationship between oblique muscle size and Bielschowsky head tilt phenomenon in clinically diagnosed superior oblique palsy. Invest Ophthalmol Vis Sci 2009;50:175-79

13. Helveston EM, Krach D, Plager DA, et al. A new classification of superior oblique palsy based on congenital variations in the tendon. Ophthalmology 1992;99:1609-15

14. Demer JL, Miller JM. Magnetic resonance imaging of the functional anatomy of the superior oblique muscle. Invest Ophthalmol Vis Sci 1995;36:906-13

15. Ozkan SB, Aribal ME, Sener EC, et al. Magnetic resonance imaging in evaluation of congenital and acquired superior oblique palsy. J Pediatr Ophthalmol Strabismus 1997;34:29-34

16. Horton JC, Tsai RK, Truwit CL, et al. Magnetic resonance imaging of superior oblique muscle atrophy in acquired trochlear nerve palsy. Am J Ophthalmol 1990;110:315-16

17. Glaser JS, Siatkowski RM. Infranuclear disorders of eye movement. In: Duane TD. Duane's Clinical Ophthalmology. Vol 2. Philadelphia: Lippincott Williams \& Wilkins; 2006 